\title{
Family stability as a moderator of the relationship between family life changes and sleep behavior
}

\author{
Jennifer Weil Malatras*, Ilana R. Luft, Karen L. Sokolowski, Allen C. Israel
}

State University of New York, Albany, USA; ${ }^{*}$ Corresponding Author: imalatras@albany.edu

Received 5 January 2012; revised 18 February 2012; accepted 16 March 2012

\begin{abstract}
The aim of the study was to evaluate the associations between aspects of family stability and sleep behavior and quality as indicators of health and well-being. Participants were 312 (166 female and 146 male) undergraduate students, ranging in age from 17 to $29(M=19.10)$. Participants' global and molecular family stability in their families of origin was assessed using the Family Life Changes Survey (FLCS) and the Stability of Activities in the Family Environment (SAFE-R), respectively. Current sleep habits and quality were measured using a modified version of the Pittsburgh Sleep Quality Inventory (PSQI). Analyses suggest family stability is related to aspects of sleep. Furthermore, it appears molecular family stability moderates the relationship between family life changes, an aspect of global family stability, and subjective sleep quality in college students. Results are important in that they suggest high levels of molecular family stability may buffer against the impact of family life changes on subjective sleep quality.
\end{abstract}

Keywords: Family Stability; Sleep; Self-Regulation

\section{INTRODUCTION}

The stability of the family environment is widely acknowledged as an important factor in development and adjustment. The work of Israel and colleagues has explored the concept of family stability and has delineated two aspects of the construct. The first aspect, termed global stability, refers to the occurrence of family life changes, such as residential moves, changes in parental employment, or changes in family structure, such as divorce, remarriage, or parental death. The term global is used to describe these events and structural changes as they are deemed more distal from an individual's daily experiences and are less easily controlled. The more proximal aspect of family stability, termed molecular family stability, refers to the predictability and consistency of daily family activities and routines [1]. This may include consistent daily routines, such as those that occur at meal or bed times, as well as activities that youth engage in with family, such as weekend activities or religious observance, or activities arranged and supported by the family but that occur without immediate family members, such as participation in extracurricular activities or time spent with friends [1,2].

Rather than conceptualizing family structure changes as single life events, Israel and colleagues' notion of global stability theorizes that such events set in motion a series of family life changes, which make it difficult to maintain a stable and predictable family environment. In this way, global and molecular family stability are related, and both have been shown to predict adjustment in both the internalizing and externalizing domains [1-6]. Family stability is also associated with the development of self-regulatory skills, including self-control [4,5].

The predictability and consistency of activities and routines within the family environment has also been shown to serve as a protective factor in the face of family adversity [2,7]. For example, molecular family stability moderates the relationship between a pessimistic attributional style and depressive symptoms, such that at high levels of family stability, pessimistic attributional style has nearly no association with depression [2]. In addition, family stability serves as a protective factor for children exposed to family psychopathology, in particular, parental depression. Family stability has been shown to protect against the influence of parental depression on children's internalizing and externalizing problems, whereby the influence of parental depressive symptoms is smallest when families are able to maintain high levels of molecular family stability [7].

Despite evidence that low levels of family stability are associated with internalizing and externalizing problems, the relationships between family stability and other indicators of adjustment, such as health behaviors, have not yet been studied. Growing evidence suggests the family environment may have a lasting impact on health. Routines and habits learned early in life, for example, are 
likely to be maintained in adulthood. In fact, Bures suggests "the social context of childhood may serve to initiate health trajectories that either protect health or increase vulnerability over the life course ([8], p. 1144)." Therefore, it seems reasonable to hypothesize that the level of family stability experienced during childhood and adolescence may be associated with health behaviors in young adulthood. The goal of the present study was to investigate the relationship between family stability and sleep behavior and quality, as one possible indicator of health behavior.

Sleep is generally thought to be an important factor in overall wellbeing, and inadequate sleep and poor sleep quality are increasingly linked with negative health outcomes, including increased risk for high blood pressure, heart disease, and other medical conditions $[9,10]$. Specifically, the quality of sleep, even more so than sleep quantity, has been found to be related to measures of health and wellbeing $[11,12]$. Furthermore, the relationship between sleep and health is thought to be bidirectional. Although the mechanisms of the transactional relationship between sleep and health are only partially understood, the immune system has been suggested as one possible mediator, with inadequate sleep associated with changes in immune cell counts, for example [13,14]. Whatever the exact mechanism, sleep problems are thought to be associated with immune functioning and health.

In addition to poor physical health outcomes, sleep problems have been related to negative psychological adjustment outcomes, as well. In a study of adolescents, Beebe and colleagues [15] tested a causal relationship between sleep restriction and adverse behavioral outcomes using a multi-night, at-home experimental sleep restriction protocol. Their findings indicate sleep restricttion induces daytime sleepiness, inattention, greater metacognitive difficulties, increased difficulties with impulse control, emotion regulation and behavioral flexibility, and increased irritability and oppositional behaviors. Research also indicates poor sleep habits and quality are associated with increased tension, irritability, depression, confusion and lower life satisfaction in college students [16-18]. Sleep has also been associated with academic performance in college, with sleep deprivation implicated in poor cognitive performance [19]. Trockel, Barnes and Egget [20], for instance, found that sleep habits, particularly wake-up times, partially accounted for variance in grade point averages.

Notably, sleep problems among college students are reportedly common. In fact, estimates suggest that more than 50 percent of university students are poor sleepers [21], and over 70 percent of students surveyed report experiencing some type of sleep difficulty on a regular basis [22]. Moreover, Buboltz, Brown and Soper suggest that college students are increasingly being recognized as "a population group particularly affected by sleep difficulties ([22], p. 131)." The factors that facilitate or interfere with sleep regulation and, in turn, impact health and well-being are complex and dynamic in nature, and various factors, including contextual factors, have been implicated.

Beginning early in life, contextual factors, particularly those associated with the family environment, are theorized to play a significant role in sleep patterns. Specifically, transactions between an infant's temperament and the caregiving environment are thought to be relevant in sleep and waking patterns [23], and the nature of parentchild interactions and certain parenting factors have been shown to predict children's sleep problems [24-26]. Research has also demonstrated a link between aspects of the family environment and adolescent sleep problems $[24,25,27]$. In fact, a recent study by Billows and colleagues [28] suggests family disorganization, characterized by inconsistent rules, irregular routines, and lack of structure, may contribute to adolescent sleep disturbance via the mechanism of sleep hygiene. The authors suggest the chaotic and unregulated lifestyle associated with disorganized families may contribute to poor sleep hygiene and quality, while greater family organization and routine may contribute to more effective sleep hygiene practices and, in turn, better sleep [28].

The influence of contextual factors on sleep behavior and quality is not limited to childhood or adolescence. Early life experiences have been shown to predict later sleep disturbances. For instance, a link between family conflict during childhood and insomnia in young adulthood has been suggested [25]. Environmental stressors may also play a more temporally proximal role in sleep difficulties. Sleep disturbances are reported in individuals who experience major stressful life events, particularly when those events are appraised as uncontrollable $[29,30]$. In fact, it has been suggested that changes associated with transition to college may lead to sleep difficulties [16]. Although major stressful life events are likely to contribute to sleep difficulties, routine also appears to play an important role. In a study of college students, lower frequency and less regularity of social rhythms, a construct similar to daily routines including activities such as getting into and out of bed, eating, and adhering to a work schedule, was found to be associated with poor sleep, while more regularity in activities was associated with good sleep [31]. Indeed, establishing positive bedtime practices, including regularly scheduled bed and wake times and consistent bedtime routines, is commonly recommended for good sleep hygiene and is considered a promising intervention for dealing with sleep problems $[10,16,32]$. In essence, both the occurrence of major life events and less regularity of routine 
may be important factors in predicting sleep problems and will likely inform interventions aimed at reducing sleep difficulties. To that end, the present study assessed sleep behavior and its relationship to the occurrence of previous life experiences, including family life events and daily routines and activities.

Specifically, the present study assessed sleep and evaluated the relationship between aspects of family stability in the family of origin and sleep behavior and quality in young adults. It was hypothesized that family life changes, one aspect of global family stability, would be associated with sleep behavior, with greater numbers of family life changes associated with poorer sleep habits and quality. Additionally, it was hypothesized that molecular family stability plays a moderating role in the relationship between family life changes and sleep behavior. At higher levels of molecular family stability, the relationship between family life changes and sleep disturbances was expected to be low in magnitude. In turn, at lower levels of molecular family stability, the relationship between family life changes and sleep disturbance was expected to be strong. Thus, molecular family stability was hypothesized to serve as a protective factor against the impact of family life changes.

\section{METHOD}

\subsection{Participants}

Participants were undergraduate students from a state university in a mid-sized northeastern city. Students participated either as part of an Introduction to Psychology course research requirement or for extra credit in another psychology course. The sample included 312 participants, $166(53.2 \%)$ female and $146(46.8 \%)$ male, ranging in age from 17 to $29(M=19.10)$. The majority identified as White $(67.5 \%)$, while $11.7 \%$ identified as Black or African American, $10.7 \%$ as Hispanic, $10.4 \%$ as Asian, $1.3 \%$ as American Indian or Alaska Native, $0.3 \%$ as Native Hawaiian or Pacific Islander, and $8.2 \%$ as other ethnicity. It is important to note participants were permitted to select more than one ethnicity, which is reflected in the fact that the ethnicity groupings sum to more than 100 percent. Finally, all but five participants reported annual income of their families prior to their entrance into college with the following breakdown: $6(2.0 \%) \$ 0$ to $\$ 14,999 ; 29(9.4 \%) \$ 15,000$ to $\$ 29,999 ; 36(11.7 \%)$ $\$ 30,000$ to $\$ 44,999 ; 40(13.0 \%) \$ 45,000$ to $\$ 59,999 ; 49$ (16.0\%) $\$ 60,000$ to $\$ 74,999 ; 53(17.3 \%) \$ 75,000$ to $\$ 89,999 ; 94(30.6 \%) \$ 90,000$ or more.

\subsection{Procedure}

All procedures followed guidelines for the protection of human subjects and were approved by the University's
Institutional Review Board. Participants were recruited using the University Psychology Department's research pool as part of a larger study. Informed consent was obtained from each participant. In a University computer lab reserved for the purpose of this study, participants, in groups of approximately eight, completed a series of measures using a computer-based survey system.

\subsection{Measures}

Participants were asked to provide information regarding their age, gender, year in school, ethnic background, and their parents' marital status, educational background and occupation, as well as their family's income. Participants also completed measures of global and molecular family stability and of sleep habits and quality.

Stability of Activities in the Family Environment $(S A F E-R)$. Molecular family stability was measured with the SAFE-R. The SAFE-R [1] is a 24-item, self-report questionnaire that assesses the level of regularity with which family activities occurred during the time in which participants were growing up. These activities include daily family routines (e.g., meal and bed time routines), as well as activities that children engage in with family (e.g., weekend activities, spending time with extended family), or activities with others that require the support of family members (e.g., extracurricular activities, spending time with friends). Participants rate items on a 7 -point scale $(0-6)$, ranging from "not at all" to "extremely" regular. Scores for all items are summed to produce a total score (SAFE-R Stability) indicating the overall degree of stability of family activities, with higher values indicating greater reported stability.

Previous research utilizing the SAFE-R has shown good internal consistency (Cronbach's alpha $=0.84)$ [1]. Test-retest reliability of the SAFE-R demonstrated a Pearson correlation coefficient of 0.86 [1]. Furthermore, validity of the SAFE-R is indicated by meaningful relationships with other family measures $[1,3]$ and by expected associations with adjustment measures $[2,4]$.

Family Life Changes Survey (FLCS). Family Life Changes were measured using the FLCS [6]. The FLCS, a 36-item, self-report questionnaire, assesses specific changes in the family of origin. Participants reported on family changes that occurred during four developmental periods (i.e., before kindergarten, elementary school, middle school, and high school). Seven family life changes are assessed, including parental absence, low parental contact, change in household residents, change in parental employment, change in residence, change in after school care, and non-normative change of school. A total family life changes score is obtained by summing the number of items for which a participant indicated the particular type of change occurred during each develop- 
mental period. The FLCS also obtains information on changes in family structure, such as parental separation, divorce, remarriage and death, and participants are asked to specify who they identified as their parental figure(s) for each developmental period. Test-retest reliability for each developmental period is reported to range from 0.90 to 0.93 [6].

Pittsburgh Sleep Quality Index (PSQI). Current sleep behaviors and quality were measured using a modified version of the PSQI [33]. The PSQI is a self-report questionnaire that assesses sleep quality and disturbances during the previous one-month interval of time. Seven component scores are generated from responses to the individual items. These components include subjective sleep quality, sleep latency, sleep duration, habitual sleep efficiency, sleep disturbances, use of sleeping medication, and daytime dysfunction. The sum of the seven components of the PSQI yields one global score. Some items were modified to be more applicable to a sample of college students, but the seven overall sleep components were maintained (a copy of the questions used in the modified version of the PSQI is available upon request). Moreover, the analyses conducted in this study focused solely on the component scores and, thus, did not utilize the global score. Test-retest reliability for each component is reported to range from 0.84 (sleep latency) to 0.65 (medication use; $p<0.001$ for each component score) [33].

\section{RESULTS}

Prior to examination of the hypotheses, the data were screened for missing data and outliers. Three cases were dropped from the sample due to missing data. Cases were removed from the sample if more than two items were missing from the SAFE-R or the PSQI. In cases where one or two items were missing from the SAFE-R, means for the subject's remaining items for that measure were imputed for the missing values [34]. Cases missing one or two items of the PSQI were retained, as they could be included in the analyses for which all necessary data were available. No cases were identified as multivariate outliers as indicated by the Mahalonobis distance (critical value, $\chi^{2}, 9 \mathrm{df}=27.877$ ). Following the data screening, 309 participants remained in the sample. Of these, $165(53.4 \%)$ were female and 144 (46.6\%) were male, and the mean age was 19.09 years.

Means and standard deviations for the SAFE-R total score, FLCS total score and the components of sleep behavior and quality are presented in Table 1. Correlations between the SAFE-R total, FLCS total and sleep component scores are presented in Table 2.

Examination of Table 2 suggests that, as expected, there was an inverse relationship between the number of
Table 1. Means and standard deviations for SAFE-R, FLCS, and sleep behavior and quality scores.

\begin{tabular}{lcccc}
\hline & N & $\begin{array}{c}\text { Possible } \\
\text { Range }\end{array}$ & Mean & $\begin{array}{c}\text { Standard } \\
\text { Deviation }\end{array}$ \\
\hline SAFE-R Total & 309 & $0-144$ & 89.87 & 19.39 \\
FLCS Total & 309 & $0-27$ & 6.75 & 4.36 \\
Subjective Sleep Quality & 308 & $0-3$ & 1.33 & 0.69 \\
Sleep Latency & 309 & $0-3$ & 0.85 & 0.88 \\
Sleep Duration & 303 & $0-3$ & 0.44 & 0.78 \\
Habitual Sleep Efficiency & 289 & $0-3$ & 0.54 & 0.82 \\
Sleep Disturbances & 309 & $0-10$ & 2.27 & 1.83 \\
Use of Sleeping Medications & 308 & $0-3$ & 0.34 & 0.76 \\
Daytime Dysfunction & 298 & $0-12$ & 3.42 & 2.32 \\
\hline
\end{tabular}

Table 2. Correlations between the SAFE-R scores, FLCS scores, and components of sleep behavior and quality.

\begin{tabular}{lcc}
\hline & FLCS Total & SAFE-R Total \\
\hline FLCS Total & - & - \\
SAFE-R Total & $-0.241^{* *}$ & - \\
Subjective Sleep Quality & $0.134^{* *}$ & $-0.207^{* *}$ \\
Sleep Latency & 0.047 & $-0.181^{* *}$ \\
Sleep Duration & 0.052 & -0.053 \\
Habitual Sleep Efficiency & 0.019 & 0.013 \\
Sleep Disturbances & 0.090 & -0.045 \\
Use of Sleeping Medication & -0.022 & $-0.144^{* *}$ \\
Daytime Dysfunction & $0.142^{* *}$ & $-0.156^{* *}$ \\
\hline
\end{tabular}

${ }^{*} p<0.05,{ }^{* *} p<0.01$.

family life changes - one aspect of global stability (FLCS) and the regularity of family activities - molecular family stability (SAFE-R). In addition, both molecular and global stability were significantly related to a number of sleep indicators.

Regression analysis supported the proposed moderation model with regard to subjective sleep quality. The interaction term between molecular family stability and family life changes explained a significant increase in variance in subjective sleep quality, $\Delta R^{2}=0.015, \Delta F(1$, $304)=4.767, p=0.030$. To provide a sense of this interaction, when molecular family stability was one standard deviation below its mean, the slope of global family stability on subjective sleep quality was 0.027 ; when molecular family stability was one standard deviation above its mean, the slope of global family stability on subjective sleep quality was -0.012 . 
This interaction is described in Table $\mathbf{3}$ and illustrated in Figure 1. The comparable interaction term with regard to "Use of Sleeping Medication" might be viewed as approaching significance $(p=0.153)$. Although this interaction term did not meet a priori criteria $(p<0.05)$, the findings may have clinical importance and, as such, may be worthy of future exploration [35]. Therewas not support for a moderation model with regard to the other sleep indicators.

\section{DISCUSSION}

As expected, multiple aspects of stability in the family of origin were related to indicators of sleep behavior and quality among young adults. With regard to molecular family stability, routines and activities in the family of origin were associated with sleep habits and quality. Specifically, lower levels of molecular stability were associated with poorer subjective sleep quality, increased sleep latency (i.e., time it takes to fall asleep), use of

Table 3. Hierarchical regression of subjective sleep quality on family stability, molecular family stability, and global family stability x molecular family stability interaction.

\begin{tabular}{ccccccc}
\hline Variable & $B$ & $S E B$ & $\beta$ & $R^{2}$ & $\Delta R^{2}$ & $\Delta F$ \\
\hline Step 1 & & & & 0.050 & 0.050 & $8.076^{* * *}$ \\
GFS & 0.014 & 0.009 & 0.089 & & & \\
MFS & -0.007 & 0.002 & $-0.185^{* *}$ & & & \\
Step 2 & & & & 0.065 & 0.015 & $4.767^{*}$ \\
GFS $\times$ MFS & -0.001 & 0.000 & $-0.130^{*}$ & & &
\end{tabular}

${ }^{*} p<0.05,{ }^{* *} p<0.01,{ }^{* * *} p<0.001$; Note: GFP = Global Family Stability; MFS $=$ Molecular Family Stability; GFS $\times$ MFS $=$ Global Family Stability $\times$ Molecular Family Stability Interaction.

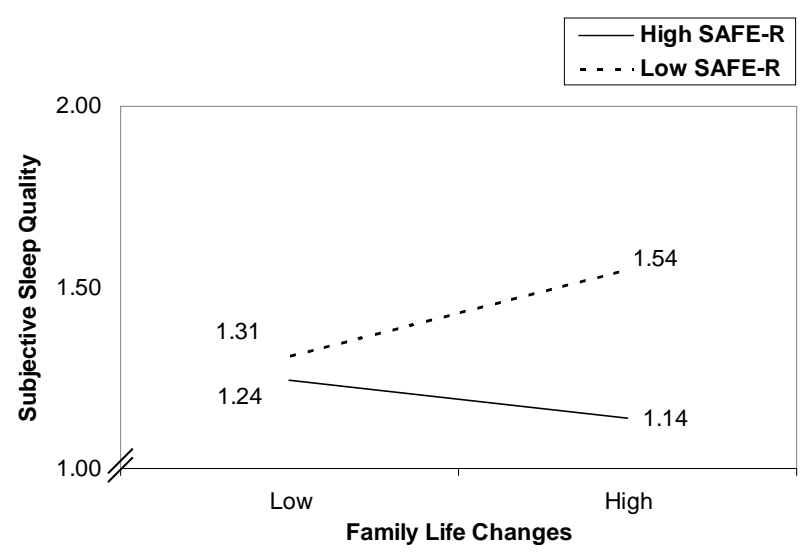

Figure 1. Slopes of family life changes on subjective sleep quality at low (1 SD below mean) and high (1 SD above mean) levels of molecular family stability (SAFE-R). Note: Subjective Sleep Quality is based on a scale from 0 to 3, with 0 indicating "very good" sleep quality and 3 indicating "very bad" sleep quality. sleeping medications, and daytime dysfunction. In addition, more family life changes (lower global stability) were associated with poorer sleep quality and daytime dysfunction. Although global stability was significantly related to important components of sleep, particularly the more distal consequences of sleep disturbance, examination of the bivariate correlations suggest that, of the two aspects of family stability, molecular stability may be more proximally related to sleep behavior and quality in young adults.

In evaluating the protective effects of molecular family stability, it was found that molecular family stability moderated the relationship between family life changes and subjective sleep quality. The adverse effects of family life changes on subjective sleep quality were strongest at low levels of molecular family stability. The data suggests high levels of molecular family stability buffered against the impact of family life changes on subjecttive sleep quality. In other words, young adults' experience of high levels of regularity in daily activities and routines in their families of origin appears to serve as a protective factor, buffering the impact of major family life changes on subjective sleep quality.

Although the present study evaluated molecular family stability as a moderator of the relationship between family life changes and sleep behavior and quality, it is possible that several mechanisms may be operating in the relationship between family stability and sleep. Disruptions in family life may set in motion a series of changes, which, in turn, negatively affect various domains of functioning, directly or indirectly affecting overall sleep quality. In other words, the relationship between family life changes and poor sleep quality may be mediated by other variables. It is possible, for instance, that molecular stability may also mediate the relationship between family life changes and sleep behavior and quality. It is plausible to hypothesize that family life changes may lead to disruptions in the regularity and predictability of routines and activities in the family, including routines associated with bed time. In turn, these disruptions may serve to interfere with sleep hygiene and overall sleep quality. Previous findings regarding the relationship between a chaotic and unregulated lifestyle and poor sleep hygiene seem to lend support to this hypothesis [28]. Yet, other variables may also play a meditational role in the relationship between family life changes and sleep behavior and quality. For instance, research supports an association between family life changes and adjustment problems [1-4]. Accordingly, it may be hypothesized that family life changes increase the likelihood of adjustment problems, such as depression or anxiety, which, in turn, contribute to the experience of sleep difficulties. As suggested by the proposed moderational model, the protective influence of molecular family stability may oper- 
ate by directly weakening the link between family life changes and sleep problems. However, it is also possible that molecular family stability attenuates the impact of family life changes on adjustment problems, in turn, moderating the relationship between adjustment and sleep difficulties.

It could also be hypothesized that molecular stability functions to facilitate youngsters' development of selfregulatory skills, which may lead to better sleep hygiene practices and sleep quality in young adulthood. Of note, the bivariate correlations suggest that low levels of molecular stability are associated with increased sleep latency and use of sleeping medications. In other words, individuals who experience low levels of regularity and predictability in daily routines and activities when growing up may be more likely, as young adults, to have difficulty falling asleep and be more likely to use sleeping medications as a way of compensating for those difficultties. Such behaviors may be suggestive of deficits in selfregulation. Israel and colleagues [36] have begun to conceptualize and have found support for viewing molecular family stability as a type of parenting behavior. As such, parents who foster molecular family stability may model self-regulatory behavior or may provide an environment that affords a level of exposure to emotional stimulation that is optimal to promoting cognitive and emotional learning and to developing self-regulatory skills and effective coping strategies. These skills may be important in regulating sleep, as well as other areas of adjustment and functioning.

Thus, the present results support a relationship between multiple aspects of family stability and sleep behavior and quality. In addition, present findings suggest that molecular stability may play a protective role in the relationship between major life events and sleep quality. Although further research is necessary to better elucidate the likely multiple and complex mechanisms involved, molecular stability in the family of origin seems to play an important role in decreasing the likelihood of sleep difficulties in young adulthood.

The findings of this study might be interpreted with caution, given that ratings of family stability were provided retrospectively and that some aspects of sleep behavior and quality, although assessed using a well-established measure, relied on response to a single item. Nevertheless, the results of this study suggest a relationship between family stability and sleep behavior and quality. The findings offer one possible explanation of the contextual factors that may contribute to sleep difficulties, which may inform efforts aimed at preventing sleep disturbances. Promoting family stability during childhood and adolescence may protect against the development of sleep problems. In addition, these findings may offer additional information and direction to clini- cians working with college students who are experienceing sleep difficulties. As mentioned previously, establishing good sleep hygiene practices is a commonly employed and often effective intervention in the treatment of sleep difficulties. Consistent with the notion of molecular family stability, sleep hygiene practices may facilitate one's ability to regulate a specific aspect of daily life, namely sleep. While establishing better sleep hygiene practices may be an effective treatment of sleep disturbances in many instances, for some individuals it may not be sufficient. Rather, interventions aimed at regulating various and multiple routines of daily life may prove to be of value and may even enhance one's ability to maintain positive sleep hygiene practices. In this way, having knowledge about an individual's experience of family stability may serve to inform clinical assessment and guide intervention. Moreover, if one is to conceptualize sleep disturbances as an indicator of adjustment, sleep problems may be indicative of more extensive difficulties in self-regulation. For these reasons, treatment aimed at facilitating general as well as more specific selfregulatory skills may serve to enhance sleep behavior and quality. In addition, efforts intended to increase the regularity of daily routines and activities, even those beyond sleep practices, may be suggested. Since sleep is considered an important component of overall health, these findings and future research may speak to the role of family stability and other contextual influences in health promotion.

\section{REFERENCES}

[1] Israel, A.C., Roderick, H.A. and Ivanova, M.Y. (2002) A measure of the stability of activities in the family environment. Journal of Psychopathology and Behavioral Assessment, 24, 85-95. doi:10.1023/A:1015336707701

[2] Ivanova, M.Y. and Israel, A.C. (2005) Family stability as a protective factor against the influences of pessimistic attributional style on depression. Cognitive Therapy and Research, 29, 243-251. doi:10.1007/s10608-005-3167-0

[3] Israel, A.C. and Roderick, H.A. (2001). A measure of the stability of family activities: An initial examination. $A s$ sessment, 8, 417-424.

[4] Sokolowski, K.L. and Israel, A.C. (2008) Perceived anxiety control as a mediator of the relationship between family stability and adjustment. Journal of Anxiety Disorders, 22, 1454-1461. doi:10.1016/j.janxdis.2008.02.009

[5] Malatras, J.W. (2009) Self-control as a mediator of the relationship between molecular family stability and adjustment. Master's Thesis, University at Albany, Albany.

[6] Israel, A.C., Sokolowski, K.L., Maruyama, S., Luft, I.R., Them, M.A., Ivanova, M.Y. and Chalmers, S.M. (2006) Assessing family life changes as an aspect of family stability. Manuscript submitted for publication.

[7] Ivanova, M.Y. and Israel, A.C. (2006) Family stability as 
a protective factor against psychopathology for urban children receiving psychological services. Journal of Clinical Child and Adolescent Psychology, 35, 564-570. doi:10.1207/s15374424jecp3504 7

[8] Bures, R.M. (2003) Childhood residential stability and health at midlife. American Journal of Public Health, 93, 1144-1148. doi:10.2105/AJPH.93.7.1144

[9] Leger, D., Schueuermaier, K., Phillip, P., Paillard, M. and Guilleminault, C. (2001) SF-36: Evaluation of quality of life in severe and mild insomniacs compared with good sleepers. Psychosomatic Medicine, 63, 49-55.

[10] National Institutes of Health (2006) In brief: Your guide to healthy sleep. National Institutes of Health Publication No. 06-5800. Accessed 9 September 2009. http://www.nhlbi.nih.gov/health/public/sleep/healthysleep fs.pdf

[11] Pilcher, J.J., Ginter, D.R. and Sadowsky, B. (1997) Sleep quality versus sleep quantity: Relationships between sleep and measures of health, well-being and sleepiness in college students. Journal of Psychosomatic Research, 42, 583596. doi:10.1016/S0022-3999(97)00004-4

[12] Pilcher J.J. and Ott, E.S. (1998) The relationship between sleep and measures of health and well-being in college students: A repeated measures approach. Behavioral Medicine, 23, 170-178. doi:10.1080/08964289809596373

[13] Motivala, S.J. and Irwin, M.R. (2007) Sleep and immunity: Cytokine pathways linking sleep and health outcomes. Current Directions in Psychological Science, 16, 21-25. doi:10.1111/j.1467-8721.2007.00468.x

[14] Savard, J., Laroche, L., Simard, S., Ivers, H. and Morin, C.M. (2003) Chronic insomnia and immune functioning. Psychosomatic Medicine, 65, 211-221. doi:10.1097/01.PSY.0000033126.22740.F3

[15] Beebe, D.W., Fallone, G., Godiwala, N., Flanigan, M., Martin, D., Schaffner, L. and Amin, R. (2008) Feasibility and behavioral effects of an at-home multi-night sleep restrict- tion protocol for adolescents. Journal of Child Psychology and Psychiatry, 49, 915-923. doi:10.1111/j.1469-7610.2008.01885.x

[16] Buboltz, W.C., Soper, B., Brown, F. and Jenkins, S. (2002) Treatment approaches for sleep difficulties in college students. Counseling Psychology Quarterly, 15, 229-237. doi:10.1080/09515070210151788

[17] Curkrowicz, K.C., Otamendi, A., Pinto, J.V., Bernert, R.A., Krakow, B. and Joiner, T.E. Jr. (2006) The impact of insomnia and sleep disturbances on depression and suicidality. Dreaming, 16, 1-10. doi:10.1037/1053-0797.16.1.1

[18] Kelly, W.E. (2004) Sleep-length and life satisfaction in a college student sample. College Student Journal, 38, 428430.

[19] Pilcher, J.J. and Walters, A.S. (1997) How sleep deprivation affects psychological variables related to college students' cognitive performance. Journal of American College Health, 46, 121-126. doi:10.1080/07448489709595597

[20] Trockel, M.T., Barnes, M.D. and Egget, D.L. (2000) Healthrelated variables and academic performance among first- year college students: Implications for sleep and other behaviors. Journal of American College Health, 49, 125131. doi:10.1080/07448480009596294

[21] Suen, L.K., Hon, L.K. and Tam, W.W.S. (2008) Association between sleep behavior and sleep-related factors among university students in Hong Kong. Chronobiology International, 25, 760-775. doi:10.1080/07420520802397186

[22] Buboltz, W.C., Brown, F. and Soper, B. (2001) Sleep habits and patterns of college students: A preliminary study. Journal of American College Health, 50, 131-135. doi:10.1080/07448480109596017

[23] Scher, A. (2008) Maternal separation anxiety as a regulator of infants' sleep. Journal of Child Psychology and Psychiatry, 49, 618-625.

[24] Adam, E.K., Snell, E.K. and Pendry, P. (2007) Sleep timing and quantity in ecological and family context: A nationally representative time-diary study. Journal of Family Psychology, 21, 4-19. doi:10.1037/0893-3200.21.1.4

[25] Gregory, A.M., Caspi, A., Moffitt, T.E. and Poulton, R. (2006) Family conflict in childhood: A predictor of later insomnia. Sleep, 29, 1063-1067.

[26] Johnson, N. and McMahon, C. (2007) Preschoolers' sleep behaviour: associations with parental hardiness, sleep-related cognitions and bedtime interactions. Journal of Child Psychology and Psychiatry, 49, 765-773.

[27] Cousins, J.C., Bootzin, R.R., Stevens, S.J., Ruiz, B.S. and Haynes, P.L. (2007) Parental involvement, psychological distress, and sleep: A preliminary examination in sleepdisturbed adolescents with a history of substance abuse. Journal of Family Psychology, 21, 104-113. doi:10.1037/0893-3200.21.1.104

[28] Billows, M., Gradisar, M., Dohnt, H., Johnston, A., McCappin, S. and Hudson, J. (2009) Family disorganization, sleep hygiene, and adolescent sleep disturbance. Journal of Clinical Child and Adolescent Psychology, 38, 745752. doi:10.1080/15374410903103635

[29] Hall, M., Buysse, D.J., Nowell, P.D., Nofzinger, E.A., Houck, P., Reynolds, C.F., et al. (2000) Symptoms of stress and depression as correlates of sleep in primary insomnia. Psychosomatic Medicine, 62, 227-230.

[30] Morin, C.M., Rodrigue, S. and Ivers, H. (2003) Role of stress, arousal, and coping skills in primary insomnia. Psychosomatic Medicine, 65, 259-267. doi:10.1097/01.PSY.0000030391.09558.A3

[31] Carney, C.E., Edinger, J.D., Meyer, B., Lindman, L. and Istre, T. (2006) Daily activities and sleep quality in college students. Chronobiology International, 23, 623-637. doi:10.1080/07420520600650695

[32] Mindell, J.A. (1999) Empirically supported treatments in pediatric psychology: Bedtime refusal and night wakenings in young children. Journal of Pediatric Psychology, 24, 465-481. doi:10.1093/jpepsy/24.6.465

[33] Buysse, D.J., Reynolds III, C.F., Monk, T.H., Berman, S.R. and Kupfer, D.J. (1989) The Pittsburgh sleep quality index: A new instrument for psychiatric practice and research. Psychiatry Research, 28, 193-213. 
doi:10.1016/0165-1781(89)90047-4

[34] Tabachnik, B.G. and Fidell, L.S. (2007) Using Multivariate Statistics. 4th Edition, Pearson Education Inc., Boston.

[35] Cohen, J. (1994) The earth is round $(\mathrm{p}<0.05)$. American Psychologist, 49, 997-1003.
doi:10.1037/0003-066X.49.12.997

[36] Chalmers, S.M. (2005) Family stability as a parenting behavior and as a mediator of the relationship between maternal sense of competence and psychosocial adjustment in children. Dissertation, University at Albany, Albany. 